\title{
Cardiorespiratory fitness does not offset the increased risk of chronic obstructive pulmonary disease attributed to smoking: a cohort study
}

\author{
Setor K. Kunutsor ${ }^{1,2,3}$ (1) $\cdot$ Sae Young Jae ${ }^{4}$ Timo H. Mäkikallio ${ }^{5,6} \cdot$ Jari A. Laukkanen ${ }^{3,7,8}$
}

Received: 22 October 2021 / Accepted: 20 December 2021 / Published online: 5 February 2022

(c) The Author(s) 2022

\begin{abstract}
Though evidence suggests that higher cardiorespiratory fitness (CRF) levels can offset the adverse effects of other risk factors, it is unknown if CRF offsets the increased risk of chronic obstructive pulmonary disease (COPD) due to smoking. We aimed to evaluate the combined effects of smoking status and CRF on incident COPD risk using a prospective cohort of 2295 middle-aged and older Finnish men. Peak oxygen uptake, assessed with a respiratory gas exchange analyzer, was used as a measure of CRF. Smoking status was self-reported. CRF was categorised as low and high based on median cutoffs, whereas smoking status was classified into smokers and non-smokers. Multivariable-adjusted hazard ratios with confidence intervals (CIs) were calculated. During 26 years median follow-up, 119 COPD cases were recorded. Smoking increased COPD risk 10.59 (95\% CI 6.64-16.88), and high CRF levels decreased COPD risk 0.43 (95\% CI 0.25-0.73). Compared with non-smoker-low CRF, smoker-low CRF was associated with an increased COPD risk in multivariable analysis 9.79 (95\% CI 5.61-17.08), with attenuated but persisting evidence of an association for smoker-high CRF and COPD risk 6.10 (95\% CI 3.22-11.57). An additive interaction was found between smoking status and CRF (RERI=6.99). Except for CRF and COPD risk, all associations persisted on accounting for mortality as a competing risk event. Despite a wealth of evidence on the ability of high CRF to offset the adverse effects of other risk factors, it appears high CRF levels have only modest attenuating effects on the very strong association between smoking and COPD risk.
\end{abstract}

Keywords Smoking $\cdot$ Cardiorespiratory fitness $\cdot$ Chronic obstructive pulmonary disease $\cdot$ Cohort study

\section{Introduction}

Chronic obstructive pulmonary disease (COPD) is a chronic inflammatory disease of the lungs that results in progressive and irreversible airflow obstruction [1]. It is the third leading cause of death globally; there were 3.23 million COPD-related deaths in 2019 [2]. Apart from being one of

Setor K. Kunutsor

skk31@cantab.net

1 National Institute for Health Research Bristol Biomedical Research Centre, University Hospitals Bristol and Weston NHS Foundation Trust and the University of Bristol, Bristol, UK

2 Musculoskeletal Research Unit, Translational Health Sciences, Bristol Medical School, University of Bristol, Learning \& Research Building (Level 1), Southmead Hospital, Bristol BS10 5NB, UK

3 Department of Medicine, Central Finland Health Care District Hospital District, Finland District, Jyväskylä, Finland the leading causes of death, COPD is associated with substantial healthcare costs and recurrent hospitalizations and also a major cause of disability-adjusted life years [1,3]. COPD represents three percent of the healthcare spending in Europe [4]. Though active smoking is the major risk factor for COPD, not all smokers develop COPD-it has been reported that $20-25 \%$ of smokers develop COPD [1]. The

4 Department of Sport Science, University of Seoul, Seoul, Republic of Korea

5 Department of Medicine, University of Helsinki, Helsinki, Finland

6 Department of Medicine, South-Karelia Central Hospital, Lappeenranta, Finland

7 Institute of Public Health and Clinical Nutrition, University of Eastern Finland, Kuopio, Finland

8 Department of Medicine, Institute of Clinical Medicine, University of Eastern Finland, Kuopio, Finland 
prevalence of COPD in non-smokers has been estimated to be $4 \%$, suggesting the existence of other risk factors [3]. Other important risk factors include occupational exposure (e.g., dust, fumes, chemicals), indoor air pollution, and infections [2,3]. Though COPD is incurable, early diagnosis and treatment can slow the progression of symptoms. In addition, COPD is potentially preventable through modulation or reduction in exposure to underlying risk factors.

Cardiorespiratory fitness (CRF), often expressed as maximal oxygen consumption $\left(\mathrm{VO}_{2} \max \right)$ in healthy individuals or peak $\mathrm{VO}_{2}$ in those with limitations to exercise, is a modifiable risk factor that can be improved through exercise training and increased physical activity [5], which is associated with reduced risk of COPD [6]. CRF is an independent predictor for all-cause and disease-specific morbidity and mortality [5]. High CRF levels have also been demonstrated to be associated a lower risk of respiratory diseases including incident COPD and death from COPD [7, 8]. CRF, which is dependent on both cardiovascular and pulmonary function, has recently been proposed as a vital sign and reported to be stronger than many traditional risk factors for COPD, such as type 2 diabetes mellitus and smoking [5]. There is growing consistent evidence that higher levels of CRF can attenuate or offset the adverse effects of other risk factors [9-11]. Furthermore, studies have demonstrated the protective effect of higher CRF against smoking-related cancer incidence and mortality $[12,13]$. Given the overall evidence, we hypothesized that CRF could offset the increased risk of COPD due to smoking. In this context, we aimed to evaluate the combined effects of smoking status and CRF on the risk of incident COPD using a population-based prospective cohort of 2295 middle-aged and older Finnish men. We also evaluated the separate associations of smoking status and CRF with the risk of COPD to confirm previous evidence of the associations.

\section{Methods}

The study population was part of the Kuopio Ischemic Heart Disease (KIHD) population-based prospective cohort study, comprising a representative sample of middle-aged and older men aged 42-61 years recruited from Kuopio, eastern Finland. They had baseline examinations performed from March 1984 through December 1989. The study was approved by the Research Ethics Committee of the University of Eastern Finland, and each participant gave written informed consent. Participants completed self-administered health and lifestyle questionnaire for the assessment of smoking and other factors. Smoking was categorised as smokers and non-smokers. A participant was defined as a smoker if he had ever smoked regularly and had smoked cigarettes, cigars, or a pipe within the past 30 days. Peak oxygen uptake $\left(\mathrm{VO}_{2 \text { peak }}\right)$ was used as a measure of CRF, which was directly assessed using a computerized metabolic measurement system (Medical Graphics, MCG, St. Paul, Minnesota) during progressive exercise testing to volitional fatigue on an electrically braked cycle ergometer [14]. The standardized testing protocol included a 3 -min warm-up at 50 watts $(\mathrm{W}) ; 1 \mathrm{~W}=6.12 \mathrm{~kg} / \mathrm{min}$ ), followed by $20 \mathrm{~W} / \mathrm{min}$ increases in workload with direct analyses of expired respiratory gases. We included all incident cases of COPD that occurred from study enrollment through 2014. No losses to follow-up were recorded in the KIHD study. Participants (using Finnish personal identification codes) are under continuous annual surveillance for the development of new outcome events. Incident COPD cases were collected by linkage to the National Hospital Discharge Register. Qualified physicians made the diagnoses of COPD which was based on clinical history, symptoms and spirometry findings (based on forced expiratory volume in $1 \mathrm{~s}$ (FEV1) and forced vital capacity (FVC)). The FEV1/FVC ratio $<0.70$ of the participants' best reading was used as the threshold for expiratory airway obstruction, i.e., COPD.

Multivariable-adjusted hazard ratios (HRs) with 95\% confidence intervals (CIs) for incident COPD were estimated using Cox proportional hazard models. Selection of confounders was based on their previously established role as risk factors for COPD, evidence from previous research, or their potential as confounders based on known associations with COPD and observed associations with the exposures using the available data [15]. CRF was modelled as both categorical (tertiles) and continuous (per standard deviation (SD) increase) variables. Evaluation of the joint association of smoking status and CRF with COPD risk was based on the following four combinations of smoking status categories and median cutoffs for CRF: non-smoker-low CRF; nonsmoker-high CRF; smoker-low CRF; and smoker-high CRF. Formal tests of interaction were used to assess if the two exposures are independent on the risk of COPD. Interactions between smoking status and CRF were examined on both the additive and multiplicative scales in relation to COPD risk. Additive interactions were assessed using the "relative excess risk due to interaction" (RERI), computed for binary variables as RERI $\mathrm{HR}_{1}=\mathrm{HR}_{11}-\mathrm{HR}_{10}-\mathrm{HR}_{01}+1$ [16]. Multiplicative interactions were assessed using the ratio of $\mathrm{HRs}=\mathrm{HR}_{11} /\left(\mathrm{HR}_{10} \mathrm{xHR}_{01}\right)$ [16]. A positive additive interaction is indicated if RERI $>0$ and a positive multiplicative interaction is indicated if the ratio of HRs $>1$. All statistical analyses were conducted using Stata version MP 17 (Stata Corp, College Station, Texas). 
Table 1 Baseline characteristics of study participants

\begin{tabular}{ll}
\hline Characteristics & $\begin{array}{l}\text { Mean (SD) or } \\
\text { median (IQR) or } \\
\mathrm{n}(\%)\end{array}$ \\
\hline Cardiorespiratory fitness, ml/kg/min & $30.3(8.0)$ \\
Age & \\
Questionnaire/prevalent conditions & \\
Age, year & $53(5)$ \\
Alcohol consumption, g/week & $31.5(6.4-92.3)$ \\
Total energy intake, kJ/day & $9919(2589)$ \\
Leisure-time physical activity, kJ/day & $1208(631-1991)$ \\
History of type 2 diabetes & $80(3.5)$ \\
Current smoking & $723(31.5)$ \\
History of CHD & $541(23.6)$ \\
History of asthma & $77(3.4)$ \\
History of chronic bronchitis & $163(7.1)$ \\
History of tuberculosis & $87(3.8)$ \\
Physical measurements & \\
BMI, kg/m ${ }^{2}$ & $26.9(3.5)$ \\
SBP, mmHg & $134(17)$ \\
DBP, mmHg & $89(10)$ \\
Socio-economic status & $8.43(4.25)$ \\
Blood biomarkers & \\
Total cholesterol, mmol/l & $5.91(1.07)$ \\
HDL-C, mmol/l & $1.29(0.30)$ \\
Fasting plasma glucose, mmol/l & $5.34(1.32)$ \\
\hline
\end{tabular}

$B M I$ body mass index, $C H D$ coronary heart disease, $D B P$ diastolic blood pressure, $H D L-C$ high-density lipoprotein cholesterol, $I Q R$ interquartile range, $S D$ standard deviation, $S B P$ systolic blood pressure

\section{Results}

The overall mean (SD) age and CRF of study participants at baseline was $53(5)$ years and $30.3(8.0) \mathrm{ml} / \mathrm{kg} / \mathrm{min}$, respectively (Table 1). Age-standardized values of CRF based on methods previously suggested [17] are provided in Appendix 1. Smokers comprised 31.5\% (723) of the study participants. During a median (interquartile range) follow-up of 26.0 (18.3-28.0) years, 119 incident cases of COPD occurred. Compared to non-smokers, smokers had an increased risk of COPD following adjustment for age, body mass index, history of type 2 diabetes, histories of coronary heart disease, asthma, chronic bronchitis and tuberculosis, alcohol consumption, energy intake, leisuretime physical activity, and socioeconomic status 11.39 (95\% CI 7.16-18.11), which was minimally attenuated to 10.59 (95\% CI 6.64-16.88) following further adjustment for CRF. A multivariable restricted cubic spline curve showed that the risk of COPD decreased continuously with increasing CRF across the range $25-65 \mathrm{ml} / \mathrm{kg} /$ $\min (P$-value for nonlinearity $=0.95)($ Fig. 1$)$. The HR for

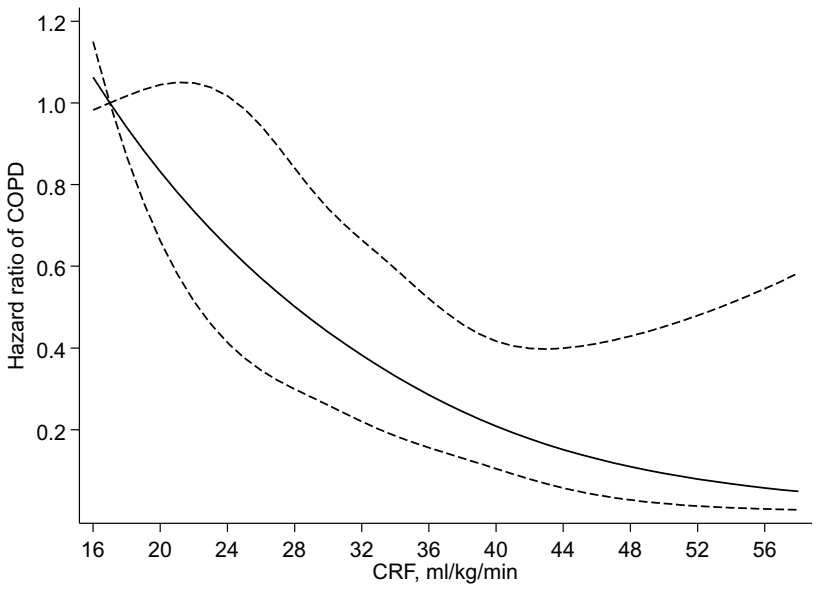

Fig. 1 Restricted cubic spline of the hazard ratios of incident chronic obstructive pulmonary disease with cardiorespiratory fitness. Reference value for cardiorespiratory fitness is $17 \mathrm{ml} / \mathrm{kg} / \mathrm{min}$; dashed lines represent the $95 \%$ CIs for the spline model (solid line). Models were adjusted for age, body mass index, history of type 2 diabetes, prevalent coronary heart disease, history of asthma, history of chronic bronchitis, history of tuberculosis, alcohol consumption, energy intake, leisure-time physical activity, and socioeconomic status. $C O P D$ chronic obstructive pulmonary disease

COPD per $1 \mathrm{SD}$ increase in CRF in analysis adjusted for the covariates above plus smoking status was 0.66 (95\% CI 0.52-0.84) (Table 2). When the top tertile of CRF was compared to the bottom tertile, the corresponding HR for COPD was 0.43 (95\% CI 0.25-0.73) (Table 2).

Crude cumulative hazard curves showed the risk for COPD was highest for smoker-low CRF group compared with other groups $(P$-value for log-rank test $<0.001$; Fig. 2). Compared with non-smoker-low CRF, smokerlow CRF was associated with an increased risk of COPD in multivariable analysis 9.79 (95\% CI 5.61-17.08), with attenuated but persisting evidence of an association for smoker-high CRF and COPD risk 6.10 (95\% CI 3.22-11.57). Results of interaction analysis showed the RERI was 6.99 and the ratio of HRs was 0.72 , indicating the presence of an additive interaction but absence of a multiplicative interaction.

Given the high mortality rate in the KIHD cohort, we included a fourth model in the association analysis to estimate the baseline cumulative subhazard of COPD considering all-cause mortality as a competing outcome to COPD. A total of 1112 deaths occurred during follow-up. In analyses including all-cause mortality as a competing risk event, there was still significant evidence of associations of smoking status and smoking status-CRF combinations (smoker-low CRF and smoker-high CRF) with 
Table 2 Separate and combined associations of smoking status and cardiorespiratory fitness with the risk of chronic obstructive pulmonary disease

\begin{tabular}{|c|c|c|c|c|c|c|c|c|c|}
\hline \multirow{2}{*}{$\begin{array}{l}\text { Exposure cat- } \\
\text { egories }\end{array}$} & \multirow[t]{2}{*}{ Events/total } & \multicolumn{2}{|l|}{ Model 1} & \multicolumn{2}{|l|}{ Model 2} & \multicolumn{2}{|l|}{ Model 3} & \multicolumn{2}{|l|}{ Model 4} \\
\hline & & HR $(95 \%$ CI $)$ & $P$-value & HR $(95 \%$ CI) & $P$-value & $\mathrm{HR}(95 \% \mathrm{CI})$ & $P$-value & HR $(95 \%$ CI $)$ & $P$-value \\
\hline \multicolumn{10}{|l|}{ Smoking status } \\
\hline Non-smoker & $25 / 1572$ & Ref. & & Ref. & & Ref. & & Ref. & \\
\hline Smoker & $94 / 723$ & $\begin{array}{l}12.57(8.07- \\
19.59)\end{array}$ & $<0.001$ & $\begin{array}{l}11.39(7.16- \\
18.11)\end{array}$ & $<0.001$ & $\begin{array}{l}10.59(6.64- \\
16.88)\end{array}$ & $<0.001$ & $\begin{array}{l}7.72(3.40- \\
17.54)\end{array}$ & $<0.001$ \\
\hline \multicolumn{10}{|l|}{$C R F(\mathrm{ml} / \mathrm{kg} / \mathrm{min})$} \\
\hline $\begin{array}{l}\text { Per } 1 \mathrm{SD} \\
\text { increase in } \\
\mathrm{CRF}\end{array}$ & $119 / 2295$ & $0.60(0.48-0.74)$ & $<0.001$ & $0.59(0.46-0.74)$ & $<0.001$ & $0.66(0.52-0.84)$ & 0.001 & $0.74(0.50-1.10)$ & 0.13 \\
\hline $\begin{array}{l}\text { Tertile } 1 \\
\quad(6.4-26.8)\end{array}$ & $62 / 765$ & Ref. & & Ref. & & Ref. & & Ref. & \\
\hline $\begin{array}{l}\text { Tertile } 2 \\
\quad(26.9-33.2)\end{array}$ & $32 / 765$ & $0.46(0.30-0.71)$ & $<0.001$ & $0.49(0.31-0.76)$ & 0.002 & $0.49(0.31-0.77)$ & 0.002 & $0.72(0.29-1.79)$ & 0.48 \\
\hline $\begin{array}{l}\text { Tertile } 3 \\
\quad(33.3-65.0)\end{array}$ & $25 / 765$ & $0.36(0.22-0.58)$ & $<0.001$ & $0.34(0.20-0.58)$ & $<0.001$ & $0.43(0.25-0.73)$ & 0.002 & $0.61(0.20-1.81)$ & 0.37 \\
\hline \multicolumn{10}{|c|}{ Smoking status and CRF $(\mathrm{ml} / \mathrm{kg} / \mathrm{min})$ combination } \\
\hline $\begin{array}{l}\text { Non-smoker-low } \\
\text { CRF }\end{array}$ & $17 / 733$ & Ref. & & Ref. & & NA & NA & Ref. & \\
\hline $\begin{array}{l}\text { Non-smoker- } \\
\text { high CRF }\end{array}$ & $8 / 839$ & $0.43(0.18-1.00)$ & 0.05 & $0.45(0.19-1.07)$ & 0.07 & NA & NA & $0.28(0.06-1.27)$ & 0.10 \\
\hline $\begin{array}{l}\text { Smoker-low } \\
\text { CRF }\end{array}$ & $60 / 415$ & $\begin{array}{l}10.11(5.88- \\
17.37)\end{array}$ & $<0.001$ & $\begin{array}{l}9.79(5.61- \\
17.08)\end{array}$ & $<0.001$ & NA & NA & $\begin{array}{l}4.89(1.82- \\
13.15)\end{array}$ & 0.002 \\
\hline $\begin{array}{l}\text { Smoker-high } \\
\text { CRF }\end{array}$ & $34 / 308$ & $\begin{array}{l}7.13(3.92- \\
12.97)\end{array}$ & $<0.001$ & $\begin{array}{l}6.10(3.22- \\
11.57)\end{array}$ & $<0.001$ & NA & NA & $\begin{array}{l}4.44(1.42- \\
13.89)\end{array}$ & 0.01 \\
\hline
\end{tabular}

$C I$ confidence interval, $C R F$ cardiorespiratory fitness, $H R$ hazard ratio, $N A$ not applicable, ref reference, $S D$ standard deviation

Model 1: adjusted for age

Model 2: model 1 plus body mass index, history of type 2 diabetes, prevalent coronary heart disease, history of asthma, history of chronic bronchitis, history of tuberculosis, alcohol consumption, energy intake, leisure-time physical activity, and socioeconomic status

Model 3: model 2 plus CRF for smoking status and smoking status for CRF

Model 4: model 3 plus all-cause mortality as a competing risk event

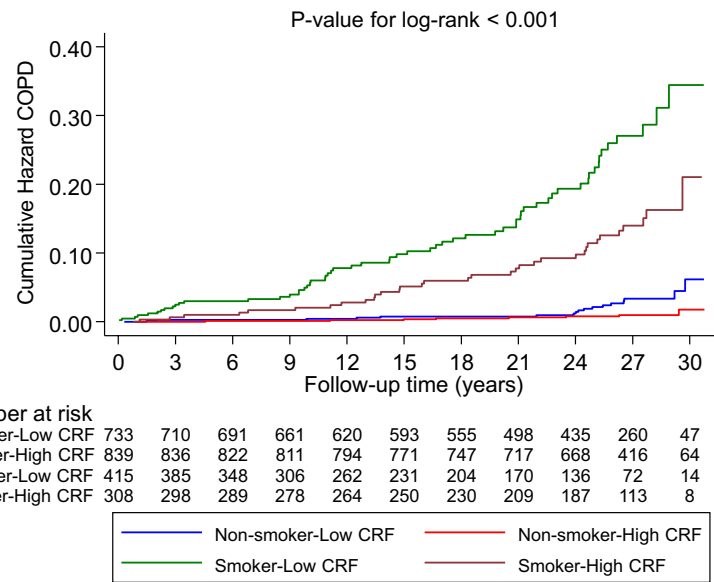

Fig. 2 Crude cumulative Kaplan-Meier curves for COPD during follow-up according to combined categories of smoking status and CRF. $C O P D$ chronic obstructive pulmonary disease, $C R F$ cardiorespiratory fitness
COPD risk, but the association was attenuated to null for CRF and COPD risk (Table 2).

To minimize the effects of potential reverse causation, we re-analysed the data on exclusion of the first five years of follow-up and the findings were similar to the main results (Appendix 2).

\section{Comment}

Our findings showed that smokers were about 11 times as likely to develop COPD than non-smokers, findings which confirm the well-established fact that smoking is the major risk factor for COPD. We have also confirmed the independent associations of elevated levels of CRF with a decreased risk of COPD, which was consistent with a linear dose-response relationship. New findings based on the joint associations of smoking status and CRF with the risk of COPD showed that the risk of COPD was substantially 
increased in men who were smokers and had low CRF levels; however, the substantial risk of COPD due to smoking was slightly attenuated but persisted in the presence of high CRF levels. Formal analysis showed significant evidence of interactive effects of smoking status and CRF (on the additive scale) on the long-term risk of COPD. Given the high mortality rate in our study cohort which might have hindered our event of interest, the association between CRF and COPD was less robust when all-cause mortality was adjusted for as a competing risk event. This was not a surprising finding as CRF was independently associated with all-cause mortality in the cohort (findings not shown). All findings remained similar on exclusion of the first five years of follow-up.

Physical fitness, a strong predictor of future health status [18], has CRF and muscular fitness as its main components [19]. High levels of CRF have well-established health benefits and the ability to modify or offset the adverse effects of other risk factors. The current findings show that high CRF levels do have interactive and attenuating effects on the association between smoking and COPD risk, but these effects are only modest. This could be due to the fact that the relationship between smoking and COPD is very strong and causal [20]. A substantial proportion of COPD cases are caused by other factors such as occupational exposure, environmental tobacco smoke, indoor air pollution, and genetic factors, but their contribution is much less than active smoking. Though the chronic inflammatory process in COPD persists after smoking cessation, the most clinically and costeffective treatment for COPD is still smoking cessation [20].

Our evaluation of the separate effects of CRF levels on COPD risk showed that high CRF levels could confer protective effects irrespective of smoking status. Taking the whole evidence together, it can be postulated that CRF has the potential to reduce the risk of COPD, but its effect is modest in the presence of active smoking. Like other adverse chronic outcomes, the beneficial effects of CRF on COPD may be exerted via the effects of regular physical activity. In addition to the anti-inflammatory effects of habitual physical activity [21], it has direct effects which include increasing the amount of ventilation in pulmonary airways and reducing lung function decline, hence preventing or delaying the onset of COPD [22].

The strengths of this study include the evaluation of the combined effects of smoking status and fitness levels, utilization of a large sample, the prospective cohort design with long follow-up, and the direct measurement of $\mathrm{VO}_{2 \text { peak }}$ using expired gas analysis, which provides a gold standard measure of CRF. We had access to a comprehensive panel of relevant confounders which allowed adequate adjustment and we were able account for the effect of reverse causation. Our limitations were mainly inherent and included the lack of granular data on smoking status which was only categorized into smokers and non-smokers, inability to generalize the findings to women and other ethnicities and the potential for biases such as residual confounding and regression dilution. Our reproducibility substudies of CRF measurements within the KIHD study show high within-person variability in CRF levels measured many years apart [23] which suggests the risk estimates observed in the current analysis are underestimated.

In conclusion, both smoking status and CRF are each independently associated with COPD risk in middle-aged and older Finnish men. Contrary to previous evidence on the ability of high CRF levels to offset the adverse effects of other chronic risk factors, high CRF levels have only modest attenuating effects on the heightened risk of COPD due to active smoking. The association between smoking and COPD risk is very strong and may only be modestly attenuated by lifestyle changes that increase CRF, such as increased exercise training and physical activity.

Supplementary Information The online version contains supplementary material available at https://doi.org/10.1007/s10654-021-00835-4.

Acknowledgements We thank the staff of the Kuopio Research Institute of Exercise Medicine and the Research Institute of Public Health, and the University of Eastern Finland, Kuopio, Finland, for the data collection in the study.

Author contribution SKK: conceptualization, methodology, data curation, formal analysis, investigation, writing-review \& editing. SYJ: conceptualization, methodology, investigation, writing - review \& editing. THM: conceptualization, methodology, writing - review \& editing. JAL: conceptualization, methodology, data curation, investigation, writing-review \& editing.

Funding This work was supported by the Finnish Foundation for Cardiovascular Research, Helsinki, Finland. SKK is funded by the NIHR Biomedical Research Centre at University Hospitals Bristol and Weston NHS Foundation Trust and the University of Bristol (BRC-121520011). The views expressed are those of the authors and not necessarily those of the NIHR or the Department of Health and Social Care. THM acknowledges support from the Päivikki and Sakari Solberg Foundation. The funders had no role in study design, data collection and analysis, decision to publish or preparation of the manuscript.

Data availability The data used for this study are available from the corresponding author upon reasonable request.

\section{Declarations}

Conflict of interest All authors declare that they have no conflict of interest to disclose.

Open Access This article is licensed under a Creative Commons Attribution 4.0 International License, which permits use, sharing, adaptation, distribution and reproduction in any medium or format, as long as you give appropriate credit to the original author(s) and the source, provide a link to the Creative Commons licence, and indicate if changes were made. The images or other third party material in this article are included in the article's Creative Commons licence, unless indicated 
otherwise in a credit line to the material. If material is not included in the article's Creative Commons licence and your intended use is not permitted by statutory regulation or exceeds the permitted use, you will need to obtain permission directly from the copyright holder. To view a copy of this licence, visit http://creativecommons.org/licenses/by/4.0/.

\section{References}

1. King PT. Inflammation in chronic obstructive pulmonary disease and its role in cardiovascular disease and lung cancer. Clin Transl Med. 2015;4(1):68. https://doi.org/10.1186/s40169-015-0068-z.

2. WHO Global Health Estimates. The top 10 causes of death. https://www.who.int/news-room/fact-sheets/detail/the-top-10causes-of-death. Accessed 15 Aug 2021.

3. Raherison C, Girodet PO. Epidemiology of COPD. Eur Respir Rev. 2009;18(114):213-21. https://doi.org/10.1183/09059180. 00003609 .

4. Vestbo J, Hurd SS, Agusti AG, et al. Global strategy for the diagnosis, management, and prevention of chronic obstructive pulmonary disease: GOLD executive summary. Am J Respir Crit Care Med. 2013;187(4):347-65. https://doi.org/10.1164/rccm. 201204-0596PP.

5. Billingsley H, Rodriguez-Miguelez P, Del Buono MG, Abbate A, Lavie CJ, Carbone S. Lifestyle interventions with a focus on nutritional strategies to increase cardiorespiratory fitness in chronic obstructive pulmonary disease, heart failure, obesity, sarcopenia, and frailty. Nutrients. 2019. https://doi.org/10.3390/nu11122849.

6. Fisher JE, Loft S, Ulrik CS, et al. Physical activity, air pollution, and the risk of asthma and chronic obstructive pulmonary disease. Am J Respir Crit Care Med. 2016;194(7):855-65. https://doi.org/ 10.1164/rccm.201510-2036OC.

7. Kunutsor SK, Laukkanen T, Laukkanen JA. Cardiorespiratory fitness and future risk of pneumonia: a long-term prospective cohort study. Ann Epidemiol. 2017;27(9):603-5. https://doi.org/ 10.1016/j.annepidem.2017.08.011.

8. Hansen GM, Marott JL, Holtermann A, Gyntelberg F, Lange P, Jensen MT. Midlife cardiorespiratory fitness and the long-term risk of chronic obstructive pulmonary disease. Thorax. 2019;74(9):843-8. https://doi.org/10.1136/thora xjnl-2018-212821.

9. Jae SY, Heffernan KS, Kurl S, et al. Cardiorespiratory fitness, inflammation, and the incident risk of pneumonia. J Cardiopulm Rehabil Prev. 2021;41(3):199-201.

10. Jae SY, Kurl S, Bunsawat K, et al. Impact of cardiorespiratory fitness on survival in men with low socioeconomic status. Eur J Prev Cardiol. 2021;28(4):450-5. https://doi.org/10.1177/20474 87319901057.

11. Kokkinos P, Faselis C, Franklin B, et al. Cardiorespiratory fitness, body mass index and heart failure incidence. Eur J Heart Fail. 2019;21(4):436-44. https://doi.org/10.1002/ejhf.1433.
12. Vainshelboim B, Lima RM, Kokkinos P, Myers J. Cardiorespiratory fitness, lung cancer incidence, and cancer mortality in male smokers. Am J Prev Med. 2019;57(5):659-66. https://doi.org/10. 1016/j.amepre.2019.04.020.

13. Sui X, Lee DC, Matthews CE, et al. Influence of cardiorespiratory fitness on lung cancer mortality. Med Sci Sports Exerc. 2010;42(5):872-8. https://doi.org/10.1249/MSS.0b013e3181 c47b65.

14. Kunutsor SK, Khan H, Laukkanen T, Laukkanen JA. Joint associations of sauna bathing and cardiorespiratory fitness on cardiovascular and all-cause mortality risk: a long-term prospective cohort study. Ann Med. 2018;50(2):139-46.

15. Groenwold RH, Klungel OH, Grobbee DE, Hoes AW. Selection of confounding variables should not be based on observed associations with exposure. Eur J Epidemiol. 2011;26(8):589-93. https:// doi.org/10.1007/s10654-011-9606-1.

16. Li R, Chambless L. Test for additive interaction in proportional hazards models. Ann Epidemiol. 2007;17(3):227-36. https://doi. org/10.1016/j.annepidem.2006.10.009.

17. Kokkinos P, Faselis C, Franklin B, et al. Cardiorespiratory fitness, body mass index and heart failure incidence. Eur J Heart Fail. 2019. https://doi.org/10.1002/ejhf.1433.

18. Kodama S, Saito K, Tanaka S, et al. Cardiorespiratory fitness as a quantitative predictor of all-cause mortality and cardiovascular events in healthy men and women: a meta-analysis. JAMA, J Am Med Assoc. 2009;301(19):2024-35. https://doi.org/10.1001/jama. 2009.681 .

19. Artero EG, Lee DC, Lavie CJ, et al. Effects of muscular strength on cardiovascular risk factors and prognosis. J Cardiopulm Rehabil Prev. 2012;32(6):351-8. https://doi.org/10.1097/HCR.0b013 e3182642688.

20. Laniado-Laborin R. Smoking and chronic obstructive pulmonary disease (COPD). Parallel epidemics of the 21 century. Int J Environ Res Public Health. 2009;6(1):209-24. https://doi.org/10.3390/ ijerph6010209.

21. Hopkinson NS, Polkey MI. Does physical inactivity cause chronic obstructive pulmonary disease? Clin Sci (Lond). 2010;118(9):565-72. https://doi.org/10.1042/CS20090458.

22. Garcia-Aymerich J, Lange P, Benet M, Schnohr P, Anto JM. Regular physical activity modifies smoking-related lung function decline and reduces risk of chronic obstructive pulmonary disease: a population-based cohort study. Am J Respir Crit Care Med. 2007;175(5):458-63. https://doi.org/10.1164/rccm. 200607-896OC.

23. Laukkanen JA, Lavie CJ, Khan H, Kurl S, Kunutsor SK. Cardiorespiratory fitness and the risk of serious ventricular arrhythmias: a prospective cohort study. Mayo Clin Proc. 2019;94(5):833-41. https://doi.org/10.1016/j.mayocp.2018.11.027.

Publisher's Note Springer Nature remains neutral with regard to jurisdictional claims in published maps and institutional affiliations. 